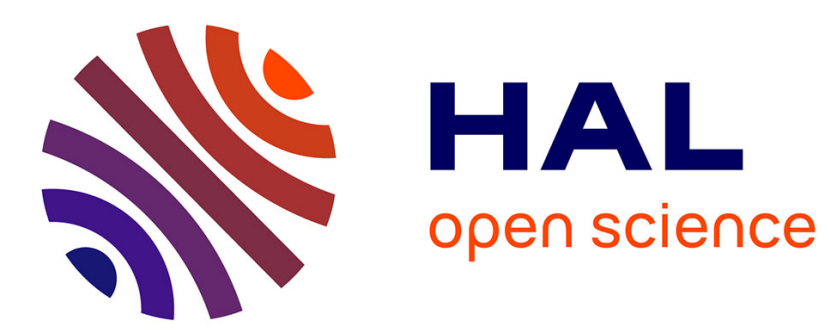

\title{
The Weakest Failure Detector to Solve the Mutual Exclusion Problem in an Unknown Dynamic Environment
}

Etienne Mauffret, Élise Jeanneau, Luciana Arantes, Pierre Sens

\section{To cite this version:}

Etienne Mauffret, Élise Jeanneau, Luciana Arantes, Pierre Sens. The Weakest Failure Detector to Solve the Mutual Exclusion Problem in an Unknown Dynamic Environment. [Technical Report] LISTIC; Sorbonne Universités, UPMC Univ Paris 06, CNRS, LIP6 UMR 7606. 2018. hal-01661127v3

HAL Id: hal-01661127

https://hal.science/hal-01661127v3

Submitted on 31 Oct 2018

HAL is a multi-disciplinary open access archive for the deposit and dissemination of scientific research documents, whether they are published or not. The documents may come from teaching and research institutions in France or abroad, or from public or private research centers.
L'archive ouverte pluridisciplinaire HAL, est destinée au dépôt et à la diffusion de documents scientifiques de niveau recherche, publiés ou non, émanant des établissements d'enseignement et de recherche français ou étrangers, des laboratoires publics ou privés. 


\title{
The Weakest Failure Detector to Solve the Mutual Exclusion Problem in an Unknown Dynamic Environment
}

\author{
Etienne Mauffret ${ }^{1}$, Denis Jeanneau ${ }^{2}$, Luciana Arantes ${ }^{2}$, and Pierre Sens ${ }^{2}$ \\ ${ }^{1}$ LISTIC/Université Savoie Mont Blanc, France \\ ${ }^{2}$ Sorbonne Université, CNRS, INRIA, LIP6, France
}

\section{Abstract}

Mutual exclusion is one of the fundamental problems in distributed computing but existing mutual exclusion algorithms are unadapted to the dynamics and lack of membership knowledge of current distributed systems (e.g., mobile ad-hoc networks, peer-to-peer systems, etc.). Additionally, in order to circumvent the impossibility of solving mutual exclusion in asynchronous message passing systems where processes can crash, some solutions include the use of $\left(\mathcal{T}+\Sigma^{l}\right)$ [3], which is the weakest failure detector to solve mutual exclusion in known static distributed systems. In this paper, we define a new failure detector $\mathcal{T} \Sigma^{l r}$ which is equivalent to $\left(\mathcal{T}+\Sigma^{l}\right)$ in known static systems, and prove that $\mathcal{T} \Sigma^{l r}$ is the weakest failure detector to solve mutual exclusion in unknown dynamic systems with partial memory losses. We consider that crashed processes may recover.

\section{Introduction}

Distributed algorithms are traditionally conceived for message-passing distributed environments which are static and whose membership is known. However, new environments such as mobile ad-hoc wireless network (MANET) or wireless sensor network (WSN), peer-topeer networks, and opportunist grids or clouds provide access to services or information regardless of node location, mobility pattern, or global view of the system. These new systems are dynamic, which means that the communication graph evolves over time, processes might join or leave the system, or crash and recover during the run. Additionally, they are unknown, i.e., processes do not initially know which other processes belong to the network, and only discover them during the run. Therefore, distributed algorithms that run on top of these new systems can not use prior distributed models for static known systems.

The mutual exclusion problem, introduced by Dijkstra in [9], is a fundamental problem in distributed computing requiring that their processes get exclusive access to one or more shared resources by executing a segment of code called critical section (CS). It specifies that, at any time, each process is either in the try, critical, exit or remainder sections. Processes cycle through these sections in order. Two processes cannot be in the critical section at the same time (safety property), and if a process is in the try section, then at some time later some process is in the critical section (liveness property).

Several mutual exclusion algorithms which tolerate process crash failures in the context of known static distributed systems have been proposed in the literature [14] [16] [1]. However, these works do not consider dynamic networks, or that a crashed process can recover. Furthermore, mutual exclusion algorithms that tolerate crashrecovery processes were mostly defined in the shared memory model, such as [12], [11], and [13], where shared variables are stored in non-volatile memory. One crashrecovery mutual exclusion algorithm for message-passing systems of which we are aware was proposed in [6] but its recovery solution works provided that failures do not occur in adjacent connected processes. Hence, the conception of mutual exclusion in unknown dynamic distributed systems where crashed processes can recover presents great challenges. 
A definition of recoverable mutual exclusion (RME) for systems with crash-recovery was presented in [12] and further studied in [11] and [13]. A main change with regard to previous definitions of fault-tolerant mutual exclusion is the critical section re-entry property, which specifies that if a process $p$ crashes while in the critical section and later recovers, then no other process may enter the critical section until $p$ re-enters it after its recovery. Intuitively, this means that the lock on the critical section is not released in the case of a temporary crash.

In this paper we consider RME on top of a message passing model, where each process has access to a volatile memory of unbounded size, which is lost after a crash and recovery, and a non-volatile memory (stable storage) of bounded size. We denote this model the partial memory loss model.

Failure detectors were introduced in [5] as a way to circumvent the impossibility to solve consensus in crashprone asynchronous systems ([10]). In [8], the $\mathcal{T}$ failure detector was shown to be the weakest failure detector to solve fault-tolerant mutual exclusion in message passing systems with a majority of correct processes. Then, in [3], the $\left(\mathcal{T}+\Sigma^{l}\right)$ failure detector was shown to be the weakest failure detector to solve the same problem with no assumption on the number of process failures.

Both of these results are restricted to known, static systems without recovery. Our paper aims to extend these results to unknown systems where crashed processes can recover, with partial memory loss.

$\left(\mathcal{T}+\Sigma^{l}\right)$ is the sum of two failure detectors. It provides two outputs, one of which verifies the properties of $\mathcal{T}$, and another one which verifies the properties of $\Sigma^{l}$. We call $\mathcal{T} \Sigma^{l}$ the detector which provides a single output verifying the properties of both $\mathcal{T}$ and $\Sigma^{l}$.

The contributions of our paper are as follows:

- A proof that $\left(\mathcal{T}+\Sigma^{l}\right)$ is equivalent to $\mathcal{T} \Sigma^{l}$;

- The definition of the $\mathcal{T} \Sigma^{l r}$ failure detector, which is equivalent to $\mathcal{T} \Sigma^{l}$ in known, static systems without recovery, and is the weakest failure detector to solve RME in our model;

- A RME algorithm that runs on top of the proposed model using the $\mathcal{T} \Sigma^{l r}$ failure detector and which tolerates crashes and recovery of processes, thus proving that $\mathcal{T} \Sigma^{l r}$ is sufficient to solve RME in our model;
- A reduction algorithm proving the necessity of $\mathcal{T} \Sigma^{l r}$ to solve RME in our model.

The rest of the paper is organized as follows: Section 2 presents our distributed system model. Section 3 provides failure detector definitions and proves the equivalence between $\left(\mathcal{T}+\Sigma^{l}\right)$ and $\mathcal{T} \Sigma^{l}$. Section 4 gives an algorithm solving mutual exclusion using $\mathcal{T} \Sigma^{l}$. Finally, Section 5 proves that $\mathcal{T} \Sigma^{l}$ is necessary to solve mutual exclusion in an unknown dynamic distributed system.

\section{Model and Problem Definition}

This section presents the distributed system model used throughout the rest of the paper and the definition of the Recoverable Mutual Exclusion (RME) problem.

\subsection{System Model}

The system is composed of a finite set of processes, denoted $\Pi$. Each process is uniquely identified. Additionally, processes are asynchronous (there is no bound on the relative speed of processes). They communicate by sending each other messages with a point-to-point SEND/RECEIVE primitive.

Communications are asynchronous (there is no bound on message transfer delay).

\section{$2.2 \quad$ Failure Model}

A process can crash (stop executing) during the run, and may recover from the crash, or not.

Each process has access to both a volatile memory and a stable storage of bounded size. After a crash and recovery, the variables in volatile memory are reset to their initial default values. As each process has access to stable storage, we say that this model deals with partial memory loss. In the rest of the paper, the names of variables in stable storage is underlined.

A process is said to be alive at time $t$ if it never stopped executing before $t$ or if it recovered since the last time it stopped executing. A process which is not alive at time $t$ is said to be crashed at time $t$.

In the traditional crash failure model, processes are grouped into faulty processes, which eventually crash, and correct processes, which never crash. However, in a 
crash-recovery model, in any run, we consider three types of processes [2]:

1) Eventually up processes, which stop crashing after some time and remain alive forever. This type also includes processes that never crash (always up).

2) Eventually down processes, which eventually crash and never recover. This type also includes processes that crashed immediately at the start of the run and never recovered (always down).

3) Unstable processes, which crash and recover infinitely often. We assume that, infinitely often, each unstable process manages to stay alive long enough to at least send a message to each other process of which it is aware.

\subsection{Connectivity Model}

The system is dynamic in the sense that the edges in the communication graph can appear and disappear during the run. In other words, at any given time instant, each edge in the graph might or might not be available. Without any further assumption, a system in which no edge is ever available would fit this model. Since nothing can be computed in such a system, additional assumptions are needed. Therefore, we assume that the following properties are verified:

- Dynamic connectivity: Every message sent by a process that is not eventually down to a process that is not eventually down is received at least once.

- Uniqueness of reception: Every message sent is received at most once.

- First in, first out: If process $p$ sends a message $m_{1}$ to $q$ and then sends $m_{2}$ to $q$, if $q$ receives $m_{2}$ then it received $m_{1}$ first.

These properties imply not only that channels are reliable, but also that each pair of processes that are not eventually down is connected infinitely often by a path over time. This means that when a process $p$ sends a message to process $q$, then there is a path from $p$ to $q$ such that at some point in the future, every edge on this path will be available in the correct order, and sufficiently long for the message to cross the edge. Note that it is not necessary that all the edges on the path to be available at the same time, and the path that a pair of processes uses to communicate is not required to be the same every time. This connectivity assumption is referred to as a Time-Varying Graph of class $\mathcal{C}_{5}$ in [4].

Our algorithms assume that the underlying SEND/RECEIVE implementation handles message forwarding and, therefore, behaves the same way that it would in a complete communication graph with reliable channels.

\subsection{Knowledge Model}

The system is unknown, i.e., processes initially have no information on system membership or the number of processes of the system, and are only aware of their own identity. The identities of other processes can only be learned through exchanging messages. More practically, each process $p$ has access to a local variable $\underline{\text { known }}_{p}$ (in stable storage) that initially contains only $p$. Eventually, $\underline{\text { known }}_{p}$ contains the set of all processes that are not eventually down. For the sake of simplicity, our algorithms do not attempt to define the $\underline{k n o w n}_{p}$ variable and simply assume that an underlying discovery algorithm eventually fills it with the necessary process identities. This is not a strong assumption, since the dynamic connectivity property ensures that all processes will be able to communicate (and therefore learn of each other's existence) infinitely often.

\subsection{Problem Definition}

We consider the Recoverable Mutual Exclusion (RME) problem, which we define in our model as follows. At any point in time, a process either (1) does not try to access the critical section and is in the remainder section, (2) attempts to access the critical section through the try section, (3) is in critical section (CS) or (4) has recently left the critical section and is in the exit section. We consider that every user is well-formed, that is that a user will go through the remainder, try, critical and exit sections in the correct order. In case of a crash and recovery, a well-formed user will restart in the critical section if it was in the critical section when it crashed, and will restart in the remainder section, otherwise (the critical section re-entry property of [11]).

A fault-tolerant mutual exclusion algorithm must provide a TRY SECTION and an EXIT SECTION procedures 
such that the following properties are satisfied:

Safety: Two distinct alive processes $p$ and $q$ can not be in CS at the same time.

Liveness: If an eventually up process $p$ stopped crashing and is in the try section, then at some time later some process that is not eventually down is in CS.

Additionally, we consider the following fairness property:

Starvation Freedom: If no process stays in its critical section forever, then every eventually up process that stopped crashing and reaches its try section will eventually enter its CS.

Note that stable storage is necessary to solve the problem. Indeed, if a process $p$ is in the critical section when all processes simultaneously crash, without stable storage there is no way for $p$ to re-enter the critical section after recovery since no process remembers that $p$ was in the critical section in the first place.

\section{$3 \quad$ Failure Detectors}

Failure detectors were introduced by Chandra and Toueg in [5] as a way to circumvent the impossibility to solve consensus in crash-prone asynchronous systems [10]. They are distributed oracles which provide unreliable information on process crashes. The information is unreliable in the sense that correct processes might be falsely suspected of having crashed, and faulty processes might still be trusted after they crashed. Different classes of failure detectors provide different properties on the reliability of the information provided to the processes.

Failure detectors are used as an abstraction of the system model assumptions.

A failure detector $\mathcal{D}_{1}$ is said to be weaker than $\mathcal{D}_{2}$ if there exists a distributed algorithm that can implement $\mathcal{D}_{1}$ using the information on failures provided by $\mathcal{D}_{2}$. Intuitively, this means that the computing power provided to the system by $\mathcal{D}_{2}$ is stronger than the computing power provided by $\mathcal{D}_{1}$. A failure detector that is sufficient to solve a given problem while being weaker than every other failure detector that can solve it, is said to be the weakest failure detector to solve that problem. It follows that the weakest failure detector to solve a problem can be implemented in any system in which the problem can be solved.

\subsection{Failure Detectors for Mutual Exclu- sion}

In [8], Delporte-Gallet et al. introduced the trusting failure detector $\mathcal{T}$ and proved that it is the weakest failure detector to solve fault-tolerant mutual exclusion in a system with a majority of correct processes. $\mathcal{T}$ provides each process $p$ with a list of trusted processes, denoted $t r_{p}$. We denote $t r_{p}^{t}$ the value of $t r_{p}$ at time $t$. We say that process $p$ trusts process $q$ at time $t$ if $q \in t r_{p}^{t}$ and that $p$ suspects $q$ at time $t$ if $q \notin t r_{p}^{t}$. The following properties must be verified.

- Eventually strong accuracy: Every correct process $p$ is eventually trusted forever by every correct process $q$, that is $\exists t: \forall t^{\prime}>t, p \in q r_{q}^{t^{\prime}}$

- Strong completeness: Every faulty process $p$ is eventually suspected forever by every correct process $q$, that is $\exists t: \forall t^{\prime}>t, p \notin q r_{q}^{t^{\prime}}$

- Trusting accuracy: For any process $p$, if there exist times $t$ and $t^{\prime}>t$ such that $q \in t q_{p}^{t}$ and $q \notin t q_{p}^{t^{\prime}}$, then $q$ is faulty.

Bhatt et al. introduce in [3] the $\Sigma^{l}$ quorum failure detector. $\Sigma^{l}$ is a variant of the $\Sigma$ quorum failure detector [7] adapted for the mutual exclusion problem. It provides each process $p$ with a quorum of process identities, denoted $q r_{p}$. Similarly to $t r_{p}$, we denote $q r_{p}^{t}$ the value of $q r_{p}$ at time $t . \Sigma^{l}$ verifies the following properties.

- Strong completeness: Every faulty process $p$ is eventually suspected forever by every correct process $q$, that is $\exists t: \forall t^{\prime}>t, p \notin q r_{q}^{t^{\prime}}$

- Live pairs intersection: If two processes $p$ and $q$ are both alive at time $t$, then for any couple of time instants $t_{1} \leq t$ and $t_{2} \leq t, q r_{p}^{t_{1}} \cap q r_{q}^{t_{2}} \neq \emptyset$.

Bhatt et al. show in [3] that $\mathcal{T}$ and $\Sigma^{l}$ used together, denoted $\left(\mathcal{T}+\Sigma^{l}\right)$, constitute the weakest failure detector to solve mutual exclusion with any number of process failures in static, known systems.

$\left(\mathcal{T}+\Sigma^{l}\right)$ provides two outputs, $t r_{p}$ and $q r_{p}$, with $t r_{p}$ verifying the properties of $\mathcal{T}$ and $q r_{p}$ verifying the properties of $\Sigma^{l}$. We call $\mathcal{T} \Sigma^{l}$ the failure detector that provides a single output $t q_{p}$ verifying the properties of both $\mathcal{T}$ and $\Sigma^{l}$. 
Theorem 1. $\left(\mathcal{T}+\Sigma^{l}\right)$ is equivalent to $\mathcal{T} \Sigma^{l}$.

Proof. $\left(\mathcal{T}+\Sigma^{l}\right)$ can be implemented using the output of $\mathcal{T} \Sigma^{l}$ : it suffices to always return the value of $t q_{p}$ as both $t r_{p}$ and $q r_{p}$. Therefore, $\left(\mathcal{T}+\Sigma^{l}\right)$ is weaker than $\mathcal{T} \Sigma^{l}$.

$\left(\mathcal{T}+\Sigma^{l}\right)$ is sufficient to solve FTME, as shown in [3]. In Section 5 of this paper, we will prove that it is possible to use RME to implement the $\mathcal{T} \Sigma^{l r}$ failure detector (defined in Section 3.2). The same reasoning and algorithms that we use in Section 5 can be used to show that it is possible to use FTME to implement $\mathcal{T} \Sigma^{l}$. It follows that $\left(\mathcal{T}+\Sigma^{l}\right)$ is sufficient to implement $\mathcal{T} \Sigma^{l}$, and as a result, $\mathcal{T} \Sigma^{l}$ is weaker than $\left(\mathcal{T}+\Sigma^{l}\right)$.

\subsection{The $\mathcal{T} \Sigma^{l r}$ Failure Detector}

The existing definition of $\mathcal{T} \Sigma^{l}$ is for static, known networks, and therefore we need to provide new definitions, suitable for unknown dynamic networks.

In an unknown system, the lack of initial information renders difficult the implementation of some failure detector properties which must apply from the start of the run, in particular the intersection property. To circumvent this problem, we make use of the $\perp$ concept introduced in [15].

Additionally, the traditional properties of $\mathcal{T} \Sigma^{l}$ are expressed in terms of correct and faulty processes. $\mathcal{T} \Sigma^{l r}$ was rewritten using the concepts of eventually up and eventually down processes instead.

The $\mathcal{T} \Sigma^{l r}$ failure detector provides each process $p$ with a set of trusted process identities, denoted $t q_{p}$, and a flag denoted $r d y_{p} . r d y_{p}$ is initially set to $\perp$ and changes to $T$ once the failure detector has gathered enough information to verify the live pairs intersection property. We denote $t q_{p}^{t}$ the value of $t q_{p}$ at time $t$, and $r d y_{p}^{t}$ the value of $r d y_{p}$ at time $t$. We say that process $p$ trusts process $q$ at time $t$ if $q \in t q_{p}^{t}$, that $p$ suspects $q$ at time $t$ if $q \notin t q_{p}^{t}$, and that process $p$ is ready at time $t$ if $r d y_{p}^{t}=\top$. The following properties must be verified.

- Eventually strong accuracy: Every eventually up process $p$ is eventually trusted forever by every process that is not eventually down.

- Strong completeness: Every eventually down process $p$ is eventually suspected forever by every process that is not eventually down.
- Trusting accuracy: For any process $p$, if there exist times $t$ and $t^{\prime}>t$ such that $q \in t q_{p}^{t}$ and $q \notin t q_{p}^{t^{\prime}}$, then $q$ is eventually down and will never be alive after $t^{\prime}$.

- Quorum readiness: Every eventually up process is eventually ready forever.

- Live pairs intersection: If two processes $p$ and $q$ are both alive at time $t$, then for any couple of time instants $t_{1} \leq t$ and $t_{2} \leq t,\left(r d y_{p}^{t_{1}}=\top \wedge r d y_{q}^{t_{2}}=\right.$ $\top) \Longrightarrow t q_{p}^{t_{1}} \cap t q_{q}^{t_{2}} \neq \emptyset$.

The eventually strong accuracy, strong completeness and trusting accuracy properties are the original properties of $\mathcal{T}$, adapted for a crash-recovery model. We call these properties the trusting properties of $\mathcal{T} \Sigma^{l r}$.

Similarly, the strong completeness and live pairs intersection properties are the original properties of $\Sigma^{l}$, adapted for our model. The new quorum readiness property, along with the $r d y_{p}$ output variable, was added to deal with the lack of initial information in an unknown system. We call these properties the quorum properties of $\mathcal{T} \Sigma^{l r}$.

Note that the strong completeness is both a trusting property and a quorum property, since both $\mathcal{T}$ and $\Sigma^{l}$ make use of this same property.

Both trusting and quorum properties apply to the same set $t q_{p}$, which is different from preexisting definitions in which $\mathcal{T}$ and $\Sigma^{l}$ are two separate oracles with separate outputs. In Section 5, we will prove that this combined version of the detector is necessary to solve RME.

Note that in a static, known system with reliable channels and prone to crash failures without recovery, $\mathcal{T} \Sigma^{l r}$ is equivalent to $\mathcal{T} \Sigma^{l}$, and therefore $\left(\mathcal{T}+\Sigma^{l}\right)$.

\section{Sufficiency of $\mathcal{T} \Sigma^{l r}$ to solve Fault-Tolerant Mutual Exclu- sion}

In this section we introduce Algorithm 1 and prove that it solves the RME in any unknown dynamic environment enriched with the $\mathcal{T} \Sigma^{l r}$ failure detector. 


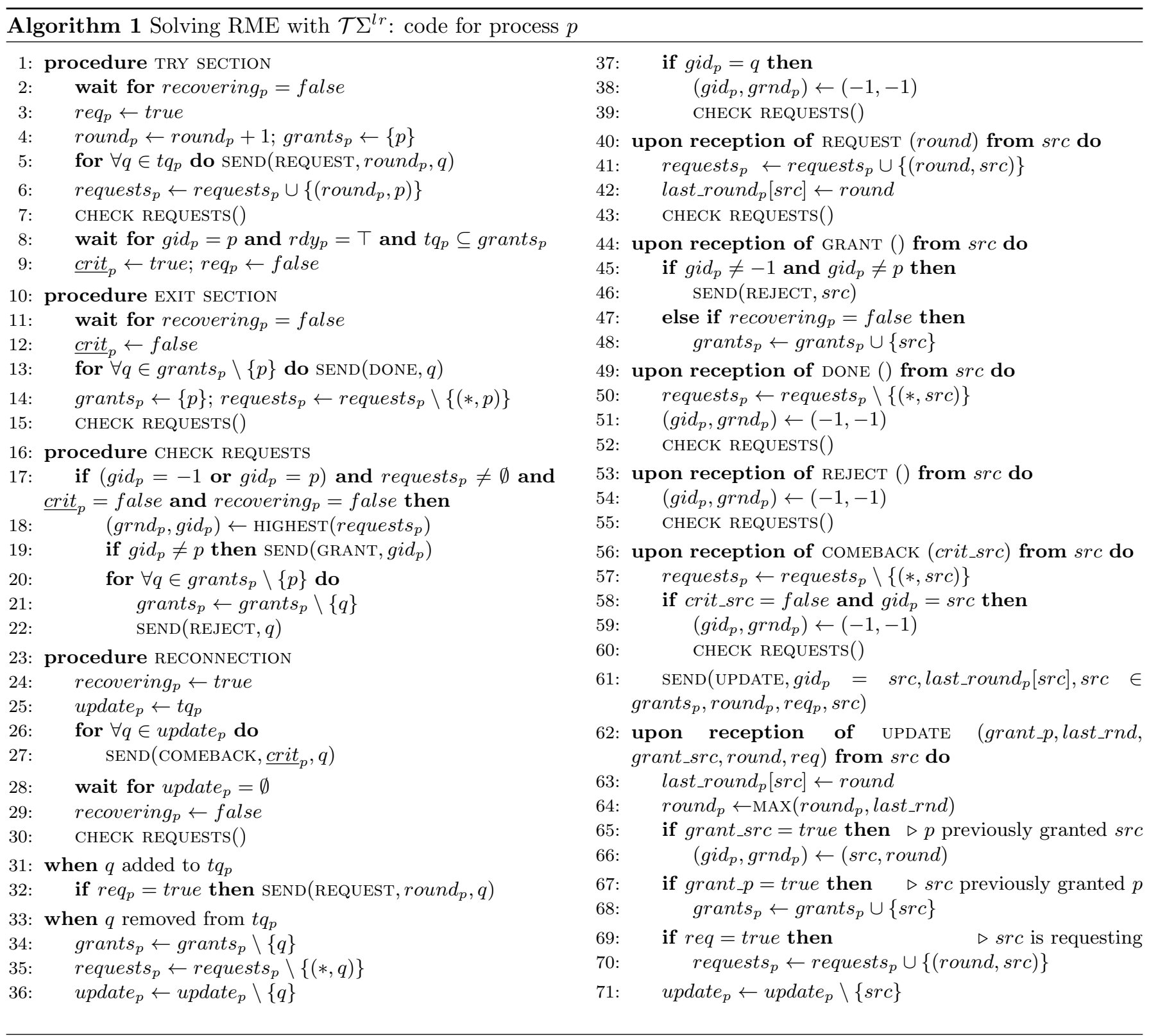




\subsection{Algorithm Description}

Let's consider $p$ the sender (source) of a message. The following types of messages are used by Algorithm 1:

REQUEST: $p$ has asked for permission to enter CS. The message contains the round number of the sender.

GRANT: $p$ has granted permission to a requesting process to enter CS.

DONE: notifies other processes that $p$ has just exited its CS.

REJECT: warns that $p$ has already given its permission to another process different from itself, thus preventing deadlocks.

COMEBACK: notifies processes that $p$ has just recovered from a crash.

UPDATE: $p$ gives information to a recently recovered process $q$ about $p$ 's requesting state, previously given permissions that $p$ granted to $q$ and vice-versa, and $q$ 's last round number of which $p$ is aware.

In Algorithm 1, each request to enter the CS, issued by $p$, is tagged by a sequence round number.

Besides having access to the output, $t q_{p}$ and $r d y_{p}$, of its local failure detector, process $p$ also keeps the following local variables, initialized with the indicated value:

crit $_{p} \leftarrow$ false: a flag indicating that $p$ is currently in CS. It is the only variable kept in stable storage. Thus, $\mathrm{crit}_{p}$ is not reinitialized after a crash and recovery.

round $_{p} \leftarrow 0$ : the local round number of $p$, which is used to number its requests. It is also used to define the current priority of $p$ to access the critical section.

last_round $_{p} \leftarrow \emptyset$ : a table associating each known process identity with its last known round number. It is used to restore the round number of other processes after they crash and recover.

$r e q_{p} \leftarrow$ false: a flag indicating that $p$ is currently in the try section.

request $s_{p} \leftarrow \emptyset$ : the set of requests received by $p$ which are pending. Each request is a couple (round,pid).

$g_{i d} \leftarrow-1$ : the identity of the last process to which $p$ granted its permission, or -1 if $p$ did not grant it. It indicates that $p$ sent a GRANT message to gid $_{p}$, and that this permission was not canceled by the reception of a DONE or REJECT message yet.

$\operatorname{grnd}_{p} \leftarrow-1$ : the current round number of the process to which $p$ granted its permission, or -1 if $p$ did not grant it. grant $_{p} \leftarrow\{p\}$ : the set of processes from which $p$ received a GRANT message.

recovering $p \leftarrow$ false: a flag indicating that $p$ is currently attempting to rebuild its volatile memory after a crash. Calls to TRY SECTION and EXIT SECTION will be delayed while recovering $g_{p}=$ false.

update $_{p} \leftarrow \emptyset$ : the set of processes from which $p$ waits for an UPDATE message. This variable is only used during the recovery phase, i.e., while recovering $g_{p}=$ true.

All of these local variables, except for $\underline{c r i t}_{p}$, are stored in volatile memory. This means that after a crash and recovery, they are reinitialized to the above default value.

Requests are totally ordered by their priority, which is defined as follows: priority $\left(\operatorname{round}_{p}, p\right)>$ $\operatorname{priority}\left(\operatorname{round}_{q}, q\right) \Leftrightarrow \operatorname{round}_{p}<\operatorname{round}_{q}$ or $\operatorname{round}_{p}=$ round $_{q}$ and $\left.p<q\right]$. The HIGHEST function takes a list of requests and returns the couple (round, $i d$ ) of the request with the highest priority among the trusted processes according to $t q_{p}$.

The CHECK REQUESTS procedure is extensively used in Algorithm 1. Provided that process $p$ did not already grant its permission to another process and is not in CS, CHECK REQUESTS compares the requests that $p$ received so far by calling the HIGHEST function (line 18), and sends a GRANT message to the process with the highest priority (line 19). In case $p$ received grants from other processes before granting its own permission, it will send REJECT messages to the processes in grants $_{p}$ in order to prevent a deadlock (lines $20-22$ ).

Whenever process $p$ wants to access the critical section, it executes the TRY SECTION: $p$ increments its round resets its grants $_{p}$ set (line 4 ), and then broadcasts a REQUEST to every process in $t q_{p}$ (line 5 ). If $p$ gets knowledge of a new process while it is still in the try section, the request will also be sent to this process (line 32 ). Process $p$ adds its own request to its requests $s_{p}$ before calling CHECK REQUESTS (lines $6-7$ ), and finally waits for permissions from every process in $t q_{p}$ (and its own permission, line 8) before entering CS.

Upon reception of a REQUEST message from process $q$ (lines $40-43$ ), process $p$ updates its knowledge about $q$ 's round number and adds the new request to its requests $s_{p}$ set. It then calls CHECK REQUESTS to decide if it should send a grant to the new requester.

When receiving a GRANT message from process $q$, if $p$ already granted its permission to some other process 
then it informs $q$ by responding with a REJECT message to prevent deadlocks (line 46). Otherwise, if $p$ is not in the recovery phase, it accepts $q$ 's permission by adding it to its grants $_{p}$ set.

Upon finishing the critical section and calling EXIT SECTION, $p$ sends to all trusted processes a DONE message (line 13). Then, $p$ resets its grants $p$ set and cancels its request (line 50) before calling CHECK REQUESTS to grant its permission to the next process.

If $p$ receives a DONE or REJECT message from process gid $_{p}$, it cancels the permission granted to gid $_{p}$ (lines 51 and 54) and calls CHECK REQUESTS. In the case of a DONE message, the request from gid $_{p}$ is also deleted from requests $_{p}$ (line 50), since gid $_{p}$ is not requesting CS anymore. However, in the case of a REJECT, the request from $g_{i d}$ is still valid and must be kept, even if it is not the highest priority request.

If $p$ crashes and recovers, the RECONNECTION procedure will be called first. This procedure initiates the recovery phase (lines $24-29$ ) by switching the recovering flag to true, which will temporarily prevent the algorithm from going into the try or exit sections (lines 2 and 11) and from sending or accepting a grant (lines 17 and 47). During the recovery phase, $p$ attempts to recover the information it lost during the crash by sending a COMEBACK message to every process in $t q_{p}$. Other processes will send UPDATE messages in response, which enables $p$ to restore its last_round, round $_{p}$, gid $_{p}$, grnd $_{p}$, and requests $s_{p}$ variables (lines $63-71$ ). The recovery phase ends when every process to which $p$ sent a COMEBACK has either responded with an UPDATE message (line 71), or crashed (line 36). After recovering, $p$ calls CHECK REQUESTS in order to choose a process to which it will grant its permission (line 30).

If $p$ receives a COMEBACK message from a process $q$, it cancels any request previously received from $q$, since a process in recovery phase can only be in the remainder or critical section (by definition of a well-formed process). If $q$ is in its remainder section $\left(\underline{c r i t}_{p}=\right.$ false), then $p$ cancels any permission it might have granted to $q$ previously (lines $58-60$ ). Finally, $p$ sends an UPDATE message to $q$.

Whenever $p$ is informed by the failure detector that a process $q$ is eventually down (lines $33-39$ ), $p$ deletes $q$

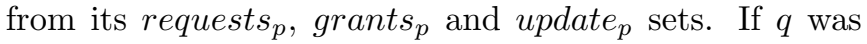
the process to which $p$ granted permission, then $p$ cancels the permission (line 38) and calls CHECK REQUESTS to grant its permission to another process, if appropriate.

\subsection{Proof of correctness}

We will prove, through the following claims, that any run of Algorithm 1 solves the RME problem.

Claim 1 (Safety). Two distinct alive processes $p$ and $q$ can not be in CS at the same time.

In order to prove Claim 1 we need to pose the following lemmata.

Lemma 1 (Uniqueness of the permission). Let $p, q_{1}, q_{2}$ be three distinct alive processes. If $p \in$ grants $_{q_{1}}$ at a time $t$ then $p$ cannot send a GRANT message to $q_{2}$ at time $t$.

Proof. The only way that $p$ can send a GRANT message to a process $q$ is on line 19 , after it selected $q$ as its gid $_{p}$. Note that the definition of the HIGHEST function also implies that $q \in t q_{p}$ at the time when the GRANT message is sent.

Suppose that $p$ has sent a GRANT message at time $t_{G}$ to another process $q_{1}$ (and therefore at time $t_{G}, g i d_{p}=q_{1}$ ).

Let us assume that there is a time $t>t_{G}$ such that $p \in$ grants $_{q_{1}}$. Let us then suppose that $p$ sends a GRANT message to another process $q_{2}$ at time $t$.

In order to send a GRANT message to $q_{2}, p$ has to set gid $_{p}$ to -1 or to $p$ at some time $t^{\prime} \in\left[t_{G}, t\right]$ (otherwise $p$ cannot pass the test on line 17). This affectation can only be done in one of the following lines:

Line 38: then $q_{1} \notin t q_{p}^{t^{\prime}}$. Since $q_{1} \in t q_{p}^{t_{G}}$, according to the trusting accuracy property of $\mathcal{T} \Sigma^{l r}, q_{1}$ has crashed at some time before $t^{\prime}$ and will never recover. It is therefore impossible that $p \in$ grants $_{q_{1}}$ at time $t$.

By resetting gid $_{p}$ to -1 after a crash. If $p$ crashed between $t_{G}$ and $t^{\prime}$, then its $g i d_{p}$ got reset to -1 . This also means that $p$ entered the recovery phase (lines $24-29$ ) at some time $t^{\prime \prime} \in\left[t_{G}, t^{\prime}\right]$. Since $q_{1} \in t q_{p}^{t_{G}}$, then according to the trusting accuracy property of $\mathcal{T} \Sigma^{l r}$, either $q_{1}$ crashed before $t^{\prime \prime}$ and will never recover (which is a contradiction), or $q_{1} \in t q_{p}^{t^{\prime \prime}}$. $p$ will therefore send a COMEBACK message to $q_{1}$ on line 27 , and $q_{1}$ will respond with a UPDATE message with the grant_src parameter set to true, which will cause $p$ to set its $g i d_{p}$ back to $q_{1}$. Since $p$ cannot have sent a GRANT message while in the recovery phase (because of the test on line 17), then $p$ cannot send the GRANT to $q_{2}$ at time $t$ which is a contradiction. 
Line 59: then $p$ received a COMEBACK message from $q_{1}$ at some time $t^{\prime \prime} \in\left[t_{G}, t^{\prime}\right]$. This means that $q_{1}$ crashed and went into the recovery phase. $p$ will respond with an update message to $q_{1}$. Since $q_{1}$ cannot leave the recovery phase until it receives $p$ 's update and because of the first in, first out property, then $p$ 's GRANT message to $q_{1}$ was received either (1) before $q_{1}$ crashed, in which case the GRANT was forgotten, or (2) during the recovery phase, in which case $q_{1}$ will ignore the GRANT because of the test on line 47 . In both cases, $p \notin$ grants $_{q_{1}}$ after $t^{\prime \prime}$, which is a contradiction.

Line 51 or 54: then $p$ received a DONE Or REJECT message from $q_{1}$ at time $t^{\prime}$. There are two cases. If $q_{1}$ sent the DONE or REJECT message after receiving the GRANT, then $q_{1}$ removed $p$ from grants $s_{1}$ on line 14 (resp. line 21) and did not add it back in afterwards, which is a contradiction. Otherwise, $q_{1}$ sent the DONE Or REJECT message before receiving $p$ 's GRANT. Since $q_{1}$ only sends DONE or REJECT messages to processes from which it previously received a GRANT, then $p$ sent another GRANT message to $q_{1}$ before $t_{G}$. This means that $p$ sent two consecutive GRANT messages to $q_{1}$ without receiving a DONE or REJECT message in between. The only way this could happen is if $p$ set its gid $_{p}$ to -1 or $p$ between sending the two GRANT messages without receiving a DONE or REJECT, which is a contradiction since this proof eliminated every other way of doing that.

Hence, we can not have $p \in$ grants $_{q_{1}}$ and $p$ sending a GRANT message to $q_{2}$ at the same time, which conclude the proof of Lemma 1 .

Lemma 2 (Self permission). Let $p, q$ be two distinct alive processes. If $p \in$ grants $_{q}$ then $p$ can not enter $C S$.

Proof. If $p \in$ grant $_{q}$, then $p$ sent a GRANT message to $q$ and therefore set its $\operatorname{gid}_{p}$ to $q$. The reasoning of the proof for Lemma 1 can be used to show that $p$ cannot change the value of its $\operatorname{gid}_{p}$ until $q$ has removed $p$ from its grants $_{q}$.

Since $p$ is required to have its $\operatorname{gid}_{p}$ set to $p$ in order to enter CS (line 8), then it is impossible for $p$ to enter CS until after $q$ removed $p$ from grants $_{q}$.

We can now prove the Claim 1 by contradiction.

Proof. Let $p_{1}, p_{2}$ be two alive, distinct processes. Let us suppose that $p_{1}$ enters CS at time $t_{1}$, and $p_{2}$ enters CS at time $t_{2}$. Let us suppose that neither process leaves CS until after the other process has entered it. According to the live pairs intersection property of $\mathcal{T} \Sigma^{l r}$, there is a process $q$ such that $q \in t q_{p_{1}}^{t_{1}} \cap t q_{p_{2}}^{t_{2}}$. It follows from the wait condition on line 8 that $q \in$ grants $_{p_{1}}$ at time $t_{1}$ and $q \in$ grant $_{p_{2}}$ at time $t_{2}$. There are two cases:

First case: $p_{1}, p_{2}$ and $q$ are all distinct. Therefore, $q$ sent a GRANT message to $p_{1}$ before $t_{1}$ and a GRANT message to $p_{2}$ before $t_{2}$. Additionally, neither process removed $q$ from their grants set before entering CS. Without loss of generality, let us assume that $q$ sent the GRANT message to $p_{1}$ first. There could be a run in which $p_{1}$ received the message immediately, and therefore added $q$ to grant $_{p_{1}}$ before $q$ sent the second GRANT to $p_{2}$. In this run, $q$ sends a GRANT message to $p_{2}$ while $q \in$ grant $_{p_{1}}$ at the same time, which is in contradiction with Lemma 1.

Second case: $q=p_{1}$ or $q=p_{2}$. Without loss of generality, let us assume that $q=p_{1}$. Since $q \in$ grants $s_{p_{2}}$ at time $t_{2}, q$ sent a GRANT message to $p_{2}$ before $t_{2}$. Since it is impossible for $q$ to send a GRANT message while in CS (because of the test on line 17), it follows that $q$ sent the GRANT before entering CS. There could be a run in which $p_{2}$ received the GRANT immediately after it was sent, therefore adding $q$ to grants $_{p_{2}}$ before $q$ entered CS, which is in contradiction with Lemma 2.

Claim 2 (Starvation freedom). If no process stays in its critical section forever, then every eventually up process that stopped crashing and reaches its try section will eventually enter its CS.

To prove the Claim 2, we pose the following lemmata:

Lemma 3 (Deadlock-free). Assuming that no process stays in CS forever, if a process $p$, which does not have the highest priority among the requesting processes, receives at least one GRANT from another process $q$, $p$ will eventually either crash forever or remove $q$ from grants $s_{p}$, and $q$ will eventually either crash forever or set gid $q$ to -1 .

Proof. Let $p$ be a process in its try section at time $t$. There exists a distinct process $p_{h}$ which is also in its try section at time $t$ and has the highest priority among requesting processes.

Let $q$ be a process distinct from $p$ that sends a GRANT message that $p$ receives at time $t$. It follows that $p$ sent a REQUEST message to $q$ at some time $t_{R}<t$.

One of the following cases applies: 
1) $p$ is eventually down, and $q$ is not. Then according to the strong completeness property of $\mathcal{T} \Sigma^{l r}, p$ will eventually be removed from $t q_{q}$ and $q$ will set gid $_{q}$ to -1 on line 38 .

2) $q$ is eventually down, and $p$ is not. Then according to the strong completeness property of $\mathcal{T} \Sigma^{l r}, q$ will eventually be removed from $t q_{p}$ and $p$ will remove $q$ from grants $_{p}$ on line 34 .

3) At time $t, \operatorname{gid}_{p} \neq-1$ and $\operatorname{gid}_{p} \neq p$. Then when $p$ receives $q$ 's GRANT message, it will never add $q$ to grant $_{p}$ and will send $q$ a REJECT message instead (line 46). When $q$ receives the REJECT message, it will set gid $_{q}$ to -1 (line 54).

4) At time $t, g_{i d_{p}}=-1$. When $p$ calls CHECK REQUESTS, it will pass the test one line 17 since requests $s_{p}$ contains at least $p$ 's request, and $\underline{\text { crit }}_{p}$ and recovering $g_{p}$ cannot be true while in CS. $p$ will then set gid $_{p}$ to something different from -1 on line 18 .

It follows from the cases above that the only way Lemma 3 could be false is if neither $p$ nor $q$ are eventually down, and $\operatorname{gid}_{p}=p$ at time $t$. Since $p$ is not eventually down, then $p$ will eventually receive $p_{h}$ 's request at some time $t^{\prime}>t$. Then one of the following cases applies:

1) During $\left[t_{R}, t^{\prime}\right], p$ does not crash, receives GRANT messages from every process in $t q_{p}$, and $r d y_{p}$ is set to $T$. Then $p$ will end the wait on line 8 and enter CS. When $p$ leaves CS, it will remove $q$ from grants $s_{p}$ on line 14 and send a DONE message to $q$ on line 13 . When $q$ receives the DONE message, it will set $g_{i d_{q}}$ to -1 on line 51 .

2) During $\left[t_{R}, t^{\prime}\right], p$ does not crash and does not receive enough GRANT messages to enter CS (or $r d y_{p}$ stays equal to $\perp$ ). Then at time $t^{\prime}$ when $p$ receives $p_{h}$ 's request, it will call CHECK REQUESTS on line 43. $p$ will pass the test on line 17 and, since $p_{h}$ is the requesting process with the highest priority, $p$ will set $g_{i d}$ to $p_{h}$. It will then remove $q$ from grants $_{p}$ on line 21 and send a REJECT message to $q$ on line 22 . When $q$ receives the REJECT message, it will set gid $_{q}$ to -1 on line 54 .

3) During $\left[t_{R}, t^{\prime}\right], p$ crashes before receiving enough GRANT messages to enter CS. When $p$ recovers, its grants $_{p}$ set is reinitialized and does not contain $q$. Since $q$ was previously in $t q_{p}$ and $q$ is not eventually down, it follows from the trusting accuracy property of $\mathcal{T} \Sigma^{l r}$ that $q$ is still in $t q_{p}$ after $p$ recovers. $p$ will therefore send a COMEBACK message to $q$ on line 27 with the crit_src parameter set to false. When $q$ receives the COMEBACK message, it will set gid $_{q}$ to -1 on line 59 . Note that because of the first in, first out property, $q$ will necessarily receive $p$ 's request before the COMEBACK message. Additionally, $p$ will receive $q$ 's GRANT message before $q$ 's UPDATE message, and will ignore the grant because of the test on line 47 .

Lemma 4 (Decreasing priority). Assuming that no process stays in the CS forever, if an unstable process $p$ is in the try section infinitely often, then the value of round increases infinitely often (and therefore, $p$ 's priority decreases infinitely often).

Proof. Let $p$ be an unstable process that is in the try section infinitely often. By definition, $p$ also crashes infinitely often. Let $q$ be any eventually up process. According to the eventually strong accuracy property of $\mathcal{T} \Sigma^{l r}, p$ will eventually trust $q$ forever.

Let $t_{0}$ be a time after which every eventually down process crashed permanently, every eventually up process stopped crashing, and $p$ started trusting $q$. According to the strong completeness property of $\mathcal{T} \Sigma^{l r}$, there is a time $t_{1} \geq t_{0}$ such that $\forall t>t_{1}, t q_{p}^{t}$ does not contain any eventually down process. Let $t_{2}>t_{1}$ be the first time after $t_{1}$ that $p$ crashes, and let $t_{3}>t_{2}$ be the first time after $t_{2}$ that $p$ enters the try section.

Every request sent by $p$ after $t_{3}$ is sent only to processes that are not eventually down, including $q$. According to the dynamic connectivity property, $q$ will receive every request sent by $p$ after $t_{3}$. Every time that $p$ crashes after $t_{3}, p$ will send a COMEBACK message to $q$. Because of the first in, first out property, $q$ will receive $p$ 's last request before receiving the COMEBACK message, and therefore when $q$ receives the COMEBACK its last_round $d_{q}[p]$ will be up to date with $q$ 's latest round $_{p}$ value from before the crash. $q$ will then respond with an UPDATE message, and $p$ will update its round $_{p}$ value on line 63 before leaving the recovery phase. As a result, crashes after $t_{3}$ do not reduce or reset $p$ 's round $_{p}$ value.

At any time $t>t_{3}$, there are three possibilities:

1) $p$ is in the exit or remainder section at time $t$. By assumption, $p$ will eventually enter the try section, and therefore increase its round $_{p}$ value on line 4 .

2) $p$ is in the CS at time $t$. Since by assumption no process stays in the section forever, $p$ will eventually leave CS and the case above applies. 
3) $p$ is in the try section at time $t$. Eventually, $p$ will either enter CS (and the case above applies), or $p$ will crash before entering the CS and therefore it will be in the remainder section after recovery (and the first case applies).

In all cases, there is a time $t^{\prime}>t$ such that round increases at time $t^{\prime}$.

Lemma 5 (Highest priority starvation freedom). Let $t$ be a time after all eventually up processes stopped crashing. Assuming that no process stays in CS forever, if an eventually up process $p$ is in the try section and has the highest priority among requesting eventually up processes at time $t$, then eventually $p$ enters $C S$.

Proof. Let $p$ be an eventually up process that is in the try section with the highest priority among requesting eventually up processes at time $t$. By contradiction, let us assume that $p$ never enters CS after $t$. It follows that $p$ will never leave the try section, since it will neither crash nor enter CS. Therefore, $p$ will never re-enter the try section and increase its round $_{p}$ value on line 4 . It follows that $p$ 's priority will never change after $t$.

Let $q_{1}$ be any unstable process. According to Lemma 4, $q_{1}$ will either eventually stop entering the try section (in which case its priority becomes irrelevant), or $q_{1}$ 's priority will be reduced infinitely often, in which case $p$ 's priority will eventually be higher than $q_{1}$ 's. As a result, there is a time $t^{\prime} \geq t$ after which $p$ has the highest priority of all requesting processes in the system.

If iid $_{p}=q_{2}$ with $q_{2}$ distinct from $q$ after $t^{\prime}$, then according to Lemma 3 , eventually $p$ will set its gid $_{p}$ to -1 and then call CHECK REQUESTS. $p$ will then set itself as $g_{i d_{p}}$ on line 18 and will never change gid $_{p}$ again.

According to the dynamic connectivity property, eventually every process in $t q_{p}$ will have received $p$ 's request. Let $q_{3}$ be any process that received $p$ 's request. If $\operatorname{gid}_{q_{3}} \neq-1$ and $\operatorname{gid}_{q_{3}} \neq q_{3}$, then after $t^{\prime}$, according to Lemma $3, q_{3}$ will eventually set gid $_{q_{3}}$ to -1 . When gid $_{q_{3}}$ is equal to -1 or $q_{3}$ after $t^{\prime}$, then $q_{3}$ will set it to $p$ on line 18 and send a GRANT message to $p$ on line 19. As a result, $p$ will receive a GRANT message from every process in $t q_{p}$.

Since $p$ is eventually up, according to the quorum readiness property of $\mathcal{T} \Sigma^{l r}$, the eventually $r d y_{p}=T$.

Finally, $p$ will pass the wait condition on line 8 and enter CS, which is a contradiction.
We can now prove Claim 2.

Proof. Let $p$ be an eventually up process that stopped crashing and is in its try section at time $t$. By contradiction, let us assume that $p$ never enters CS after $t$. It follows that $p$ will never leave the try section, since it will neither crash nor enter CS. Therefore, $p$ will never re-enter the try section and increase its round $_{p}$ value on line 4. It follows that $p$ 's priority will never change after $t$, and that every requesting unstable process will eventually have a lower priority than $p$.

Let $Q$ be the set of all requesting eventually up processes with higher priority than $p$. Let $q$ be the process in $Q$ with the highest priority. It follows from Lemma 5 that eventually, $q$ will enter CS. After $q$ leaves CS, it will either (1) stop requesting forever (and therefore leave $Q$ ) or (2) enter the try section again and therefore decrease its priority. By induction, $q$ will eventually not have the highest priority amongst requesting processes anymore, and another process in $Q$ will take its place. As a result, eventually $Q$ will become empty since every process in it will either stop requesting or increase its priority infinitely often.

Finally, $p$ will become the requesting eventually up process with the highest priority, and according to Lemma 5, will enter CS, which is a contradiction.

Claim 3 (Liveness). If an eventually up process $p$ stopped crashing and is in the try section, then at some time later some process that is not eventually down is in CS.

Proof. Let $p$ be an eventually up process that stopped crashing and is in the try section. There are two possibilities:

- Some process eventually stays in CS forever. In this case, liveness is ensured.

- Otherwise, according to Claim 2, $p$ will eventually enter CS, thus ensuring liveness.

From Claim 1 and Claim 3 we can deduce the following theorem:

Theorem 2 (Correctness). The Algorithm 1 solves the $R M E$ using $\mathcal{T} \Sigma^{l r}$ in any unknown dynamic environment. 
Corollary 1 (Sufficiency). The $\mathcal{T} \Sigma^{l r}$ failure detector is sufficient to solve the RME in any unknown dynamic environment with partial memory loss.

\section{$5 \quad$ Necessity of $\mathcal{T} \Sigma^{l r}$ to solve Fault- Tolerant Mutual Exclusion}

In this section we prove that the $\mathcal{T} \Sigma^{l r}$ failure detector is necessary to solve the RME problem in any unknown dynamic system with partial memory loss. For this purpose, we assume that there is an unknown dynamic system model $\mathcal{M}_{\mathrm{RME}}$ with partial memory loss, in which RME can be solved with some algorithm $\mathcal{A}_{\mathrm{RME}}$. We will then show that the properties of $\mathcal{T} \Sigma^{l r}$ can be implemented in $\mathcal{M}_{\text {RME }}$.

Although the purpose of this section is to show that $\mathcal{T} \Sigma^{l r}$ can be implemented with RME in $\mathcal{M}_{\mathrm{RME}}$, the same arguments and algorithms used here can also be used to show that $\mathcal{T} \Sigma^{l}$ can be implemented with fault-tolerant mutual exclusion in a static, known system without recovery. As a result, this section is also a part of the proof for Theorem 1.

The following proof is inspired by the proofs for the necessity of $\mathcal{T}$ and $\Sigma^{l}$ in [8] and [3], respectively. The main additional challenge is to merge the two proofs, since both trusting and quorum properties must apply for a same set $t q_{p}$.

The proof uses two algorithms, both of which share the following local variables:

trust $_{p} \leftarrow\{p\}$ is the set of all processes that process $p$ has heard of and that it does not suspect. This variable is in stable storage.

start $_{p} \leftarrow$ false is a flag used to delay the start of the RME algorithm.

Firstly, we introduce the algorithm $\mathcal{B}_{\mathrm{RME}}$. $\mathcal{B}_{\mathrm{RME}}$ has exactly the same code as $\mathcal{A}_{\mathrm{RME}}$, except that every call to the SEND primitive is replaced by a call to $\mathcal{B}_{\mathrm{RME}}$-SEND, as defined in Algorithm 2.

Algorithm 2 serves two purposes: (1) by using $\underline{\text { trust }}_{p}$, it enables $p$ to keep track of which processes it heard of while trying to access CS; (2) by using $s_{\text {tart }}$, it enables $p$ to delay the start of the RME algorithm.

Lemma 6. Provided that each eventually up process $p$ eventually sets start $p$ to true, Algorithm $\mathcal{B}_{\mathrm{RME}}$ solves the RME problem in $\mathcal{M}_{\mathrm{RME}}$.

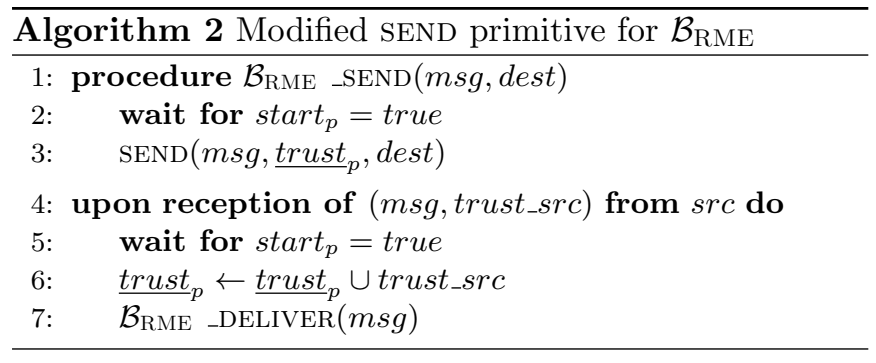

Proof. The only difference between $\mathcal{A}_{\mathrm{RME}}$ and $\mathcal{B}_{\mathrm{RME}}$ that could prevent $\mathcal{B}_{\mathrm{RME}}$ from solving RME is the wait on lines 2 and 5 . A process that never sets start $p$ to true cannot participate in the algorithm. By assumption, this is only a problem for processes that are not eventually up. If a process never sets start ${ }_{p}$ to true, then for the purpose of $\mathcal{B}_{\mathrm{RME}}$, that process behaves exactly as an always down process would behave in a run of $\mathcal{A}_{\mathrm{RME}}$.

We can now introduce Algorithm 3, which makes use of $\mathcal{A}_{\mathrm{RME}}$ and $\mathcal{B}_{\mathrm{RME}}$ to implement the properties of $\mathcal{T} \Sigma^{l r}$.

In addition to $\underline{\text { trust }}_{p}$ and start $_{p}$, Algorithm 3 uses following local variables:

known $_{p} \leftarrow\{p\}$ : as discussed in Section 2, known $_{p}$ represents the knowledge that $p$ has of other processes in the system. The algorithm does not show how known $_{p}$ is kept up to date, but simply expects that $\underline{\text { nnown }}_{p}$ will eventually contain the process identities of (at least) all eventually up processes.

$\mathrm{crash}_{p} \leftarrow \emptyset$ : the set of all processes that $p$ is certain have crashed forever. Note that this variable is in stable storage.

$t q_{p} \leftarrow \emptyset$ : the output of the $\mathcal{T} \Sigma^{l r}$ failure detector, which verifies the trusting and quorum properties.

$r d y_{p} \leftarrow \perp$ : the other output variable of $\mathcal{T} \Sigma^{l r}$, which verifies the quorum properties.

$\underline{\text { waitlist }}_{p} \leftarrow \emptyset$ : the set of processes to which $p$ must grant permission for CS. This is used to ensure starvation freedom. Note that this variable is in stable storage.

donelist $_{p} \leftarrow \emptyset$ : the set of processes to which $p$ already granted permission for CS. It prevents $p$ from always being passed over for CS access.

Algorithm 3 initially starts two tasks in parallel: TASK 1 and TASK 2. Later on, whenever process $p$ gets knowledge of a process $q$, it starts a new task for $q$ (denoted TASK $3+q)$. 


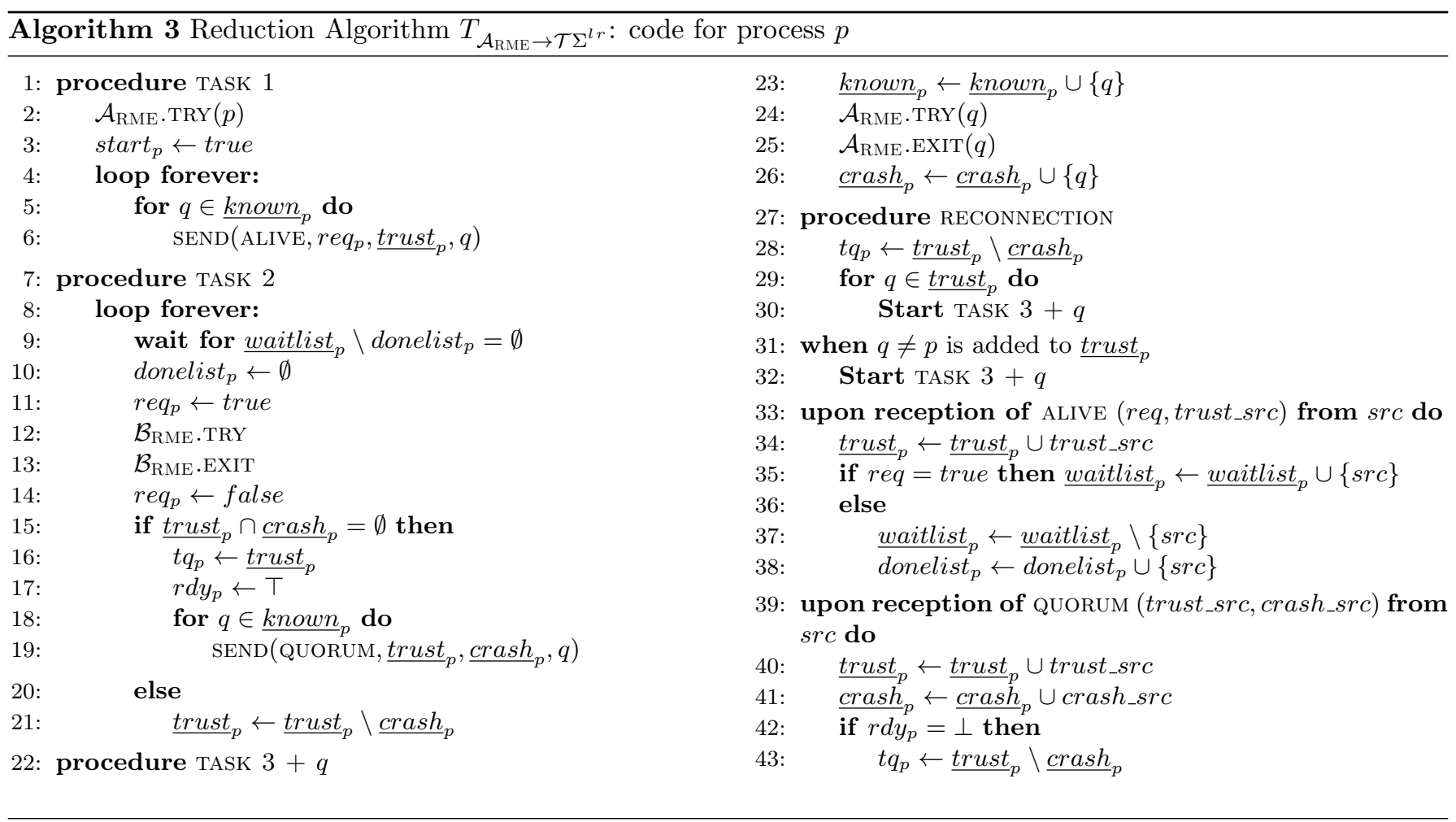


Each process $p$ has its own CS, which is handled by algorithm $\mathcal{A}_{\mathrm{RME}}$ and accessed with $\mathcal{A}_{\mathrm{RME}} \cdot \mathrm{TRY}(p)$. Additionally, there is a global CS which is handled by algorithm $\mathcal{B}_{\mathrm{RME}}$ and accessed with $\mathcal{B}_{\mathrm{RME}}$.TRY.

In TASK 1, $p$ enters its own CS and then never leaves it. Since in this case a well-formed process restarts in the CS after a recovery, this means that a recovering process will restart TASK 1 directly after line 2 if it previously managed to enter its own CS. This enables other processes to detect $p$ 's failure if it crashes permanently (if another process manages to access $p$ 's CS in TASK $3+p$, it means that $p$ crashed forever). In TASK $1, p$ also sends information to the rest of the system about its own identity and whether or not $p$ is trying to access the global CS. These ALIVE messages are used by other processes to keep $\underline{\text { trust }}_{p}, \underline{\text { waitlist }}_{p}$, and donelist $p$ up to date.

In TASK 2, $p$ tries infinitely often to access the global CS. The wait on line 9 helps to ensure that the global CS starvation freedom property is satisfied. After entering and leaving the global CS, if $p$ entered it using only messages from processes that are not crashed (test on line 15), then $p$ updates its $\mathcal{T} \Sigma^{l r}$ output variables and informs other processes with QUORUM messages. However, if $p$ used information from crashed processes to enter CS, it removes them from its $\underline{\text { trust }}_{p}$ set.

TASK $3+q$ is started by $p$ when $q$ is added to $\underline{\text { trust }}_{p}$, and is used to detect $q$ 's permanent crash.

When a process $p$ receives a QUORUM message, it updates its local $\underline{\text { trust }}_{p}$ and $\underline{\text { crash }}_{p}$ information and, if $r d y_{p}$ is currently $\perp$ (and therefore $p$ is not currently trying to verify the live pairs intersection property), then $p$ updates its $t q_{p}$.

Lemma 7 (Starvation freedom). Every eventually up process passes the lines 12 - 13 infinitely often.

Proof. By contradiction, let us assume that there is an eventually up process $p$ which does not go through CS infinitely often. There are two ways this could happen: $p$ is either stuck in the wait on line 9 forever, or $p$ is stuck in try section on line 12 forever.

First let us assume that $p$ is stuck in try section forever. Since the liveness property of RME is verified, and since no process can stay in CS forever (since the CS has no code), it follows that there is a process $q$ that enters CS infinitely often.

Eventually, $p \in \underline{\text { known }}_{q}$ and $q \in \underline{\text { known }}_{p}$. Since $p$ set $r e q_{p}$ to true on line 11 , then eventually $q$ will receive an ALIVE message from $p$ with req set to true, and $q$ will add $p$ to $\underline{\text { waitlist }}_{q}$. Because of the first in, first out property, $q$ will eventually stop receiving any ALIVE message from $p$ that has the $r e q$ value set to false. Since $q$ passes the line 10 infinitely often, eventually $p \notin$ donelist. Since $p \in$ waitlist $_{q} \backslash$ donelist $_{q}$, then eventually $q$ will wait forever on line 9 , which is a contradiction.

Now let us assume that $p$ is stuck on line 9 forever. Let $W$ be the set of processes that stay in $\underline{\text { waitlist }}_{p} \backslash$ donelist $_{p}$ for infinitely long. Note that a process $q$ that is not stuck forever in the try section on line 12 would have their req set to false and therefore would send an ALIVE message to $p$ with req set to false, and would be removed from

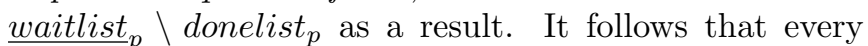
$q \in W$ is stuck forever on line 12 . If $q$ is eventually down, it eventually crashes forever and therefore cannot be in $W$. If $q$ is eventually up, according to the previous paragraph it eventually enters CS and therefore cannot be in $W$. If $q$ is unstable, it eventually crashes and resets its $r e q_{q}$ to false, and therefore cannot be in $W$. As a result, $W$ is empty and $p$ eventually ends the wait on line 9 .

Lemma 8 (Crashed completeness). A process can only be added to $\underline{\text { crash }}_{p}$ if it crashed forever.

Proof. A process can only be added to $\underline{c r a s h}_{p}$ on lines 26 and 41. In order for $p$ to add a process to $\underline{\text { crash }}_{p}$ on line 41, some other process $q$ must have added it to $\underline{\operatorname{crash}}_{q}$ on line 26 first.

In order for $p$ to add a process $q$ to $\underline{\operatorname{crash}}_{p}$ on line 26 , $p$ must first have started TASK $3+q$. This can only happen if $p$ added $q$ to $\underline{t r u s t}_{p}$. A process can be added to $\underline{\text { trust }}_{p}$ on lines 34 and 40 , or by receiving information from $q$ as part of algorithm $\mathcal{B}_{\mathrm{RME}}$. If $q$ sent a QUORUM message, then it must have passed the CS on lines 12 13 and therefore sent or received information as part of algorithm $\mathcal{B}_{\mathrm{RME}}$, which means that start $q$ was set to true. Whether $q$ set start $_{q}$ to true on line 3 or sent an ALIVE message on line 6 , it had to enter its own CS on line 2 first.

Since $q$ entered its own CS before $p$ started TASK $3+$ $q$ and will never leave it, the only way that $p$ can reach line 26 and add $q$ to $\underline{\operatorname{crash}}_{p}$ is if $q$ crashed forever. 
Claim 4 (Strong completeness). Algorithm 3 ensures the strong completeness property of $\mathcal{T} \Sigma^{l r}$ in $\mathcal{M}_{\mathrm{RME}}$.

Proof. Let $p$ be an eventually down process, and $q$ be a process that is not eventually down. Note that by construction, a process can never be added to $t q_{q}$ without being added to $\underline{\text { trust }}_{q}$ first. There are two cases:

$p$ was never added to $\underline{\text { trust }}_{q}$. Then the property is immediately verified.

$p$ was added to $\underline{\text { trust }}_{q}$. Let $r$ be some eventually up process. Eventually, $q$ will send an ALIVE message to $r$ which contains $\underline{\text { trust }}_{q}$. Therefore, $r$ will eventually add $p$ to its $\underline{\text { trust }}_{r}$ and will then start TASK $3+p$. After $p$ crashes forever, eventually $r$ will reach line 26 and add $p$ to $\underline{\text { crash }}_{r}$.

Let $t_{1}$ be a time after which all eventually down processes have crashed. Let $t_{2} \geq t_{1}$ be a time after which there are no more messages sent by eventually down processes in the system. After $t_{2}$, neither $q$ nor $r$ will ever add an eventually down process into their trust set again. According to Lemma 7, $r$ will then eventually remove all eventually down processes from $\underline{\text { trust }}_{r}$ on line 21 . Since according to Lemma 8 only eventually down processes can be in $\underline{\text { rrash }_{r}}$, after this time $r$ will always pass the test on line 15 and therefore $r$ will send a QUORUM message to $q$ infinitely often.

If $q$ goes through the loop in TASK 1 infinitely often, it will act like $r$ and eventually never have $p$ in its $t q_{q}$. If $q$ is unstable and does not go through the loop in TASK 1 infinitely often, then after it stops going through the loop it will crash and reset its $r d y_{q}$ to $\perp$. Then, the next time that $q$ receives a QUORUM message from $r$, it will add $p$ to $\underline{\mathrm{crash}}_{q}$ and remove it from $t q_{q}$ on line 43

Claim 5 (Eventually strong accuracy). Algorithm 3 ensures the eventually strong accuracy property of $\mathcal{T} \Sigma^{l r}$ in $\mathcal{M}_{\mathrm{RME}}$.

Proof. Let $p$ be an eventually up process, and $q$ a process that is not eventually down. Eventually, $q \in$ known $_{p}$. According to the liveness property of RME, $p$ will eventually enter its own CS and send an ALIVE message to $q$ on line 6 . When $q$ receives the message, it will add $p$ to its trust $_{q}$ set on line 34. It follows from Lemma 8 that $p$ will never be in $\underline{\text { rrash }}_{q}$. According to the proof for Claim $4, q$ will update its $t q_{p}$ infinitely often with $\underline{\text { trust }}_{q}$, either on line 16 or on line 43 . As a result, $p \in t q_{q}$ forever.
Claim 6 (Trusting accuracy). By construction, the only way that a process can be removed from $t q_{p}$ is by being added to $\underline{\text { crash }}_{p}$. The proof then follows directly from Lemma 8.

Claim 7 (Quorum readiness). Algorithm 3 ensures the quorum readiness property of $\mathcal{T} \Sigma^{l r}$ in $\mathcal{M}_{\mathrm{RME}}$.

Proof. Let $p$ be an eventually up process. According to the proof for Claim 4, $p$ will pass the test on line 15 infinitely often. After $p$ stops crashing, the next time it reaches line 17 , it will set $r d y_{p}$ to $\top$ forever.

Lemma 9 (Message reception intersection). Let $p_{1}$ and $p_{2}$ be two processes that enter the $C S$ of $\mathcal{B}_{\mathrm{RME}}$ at time $t_{1}$ (resp. $t_{2}$ ). Let $Q_{1}$ (resp. $Q_{2}$ ) be the set of all processes from which $p_{1}$ (resp. $p_{2}$ ) received information from (directly or through forwarding) since the last time it entered the try section before $t_{1}$ (resp. $t_{2}$ ). Then either one of the process crashed permanently before the other entered $C S$, or $Q_{1} \cap Q_{2} \neq \emptyset$.

Proof. By contradiction, let us assume that $Q_{1} \cap Q_{2}=\emptyset$.

First let us assume that in $\mathcal{B}_{\mathrm{RME}}$, a process $r$ might send a message to a process $s$ to authorize $s$ to enter CS before $s$ has entered the try section. In this case, it is possible that every process in the system would send such a message to $s$ before $s$ enters the try section. Let us now consider a run in which a process $s^{\prime}$ different from $s$ later enters the try section. If $\mathcal{B}_{\mathrm{RME}}$ allows some process to authorize $s^{\prime}$, then all other processes might do the same thing. As a result, if $s$ is not permanently crashed, $s$ and $s^{\prime}$ might enter CS at the same time, thus violating the safety property. If $\mathcal{B}_{\mathrm{RME}}$ does not allow any process to authorize $s^{\prime}$, then $s$ might never enter the try section, thus violating the liveness property. It follows that in $\mathcal{B}_{\mathrm{RME}}$, only messages received after entering the try section can authorize a process to enter CS.

Let us now consider a run in which every message between $Q_{1}$ and $Q_{2}$ is delayed until after both $p_{1}$ and $p_{2}$ have left CS. This means that the system is partitioned, and therefore algorithm $\mathcal{B}_{\mathrm{RME}}$ cannot possibly prevent a run in which both $p_{1}$ and $p_{2}$ enter CS at the same time, thus violating the safety property of RME.

Claim 8 (Live pairs intersection). Algorithm 3 ensures the live pairs intersection property of $\mathcal{T} \Sigma^{l r}$ in $\mathcal{M}_{\mathrm{RME}}$. 
Proof. The live pairs intersection property only applies when $r d y_{p}$ is set to $T$, and the only way to set $r d y_{p}$ to $\top$ is on line 17 . Since lines 28 and 43 can only be reached when $r d y_{p}$ is set to $\perp$, it follows that at any time $r d y_{p}$ is equal to $T$, the current value of $t q_{p}$ was set on line 16 .

Note that $t q_{p}$ is set from $\underline{\text { trust }}_{p}$ on line 16 after $p$ recently went through the global try, critical, and exit sections with $\mathcal{B}_{\mathrm{RME}}$ on lines $12-13$. By construction, every process from which $p$ received information (even indirectly) in $\mathcal{B}_{\mathrm{RME}}$ since last entering the try section is in trust $_{p}$ at that time. Observe also that the only way to remove a process identity from $\underline{\text { trust }}_{p}$ is on line 21 , which cannot be reached between lines 12 and 16 .

Let $p_{1}$ and $p_{2}$ be two processes, and let $t$ be some time at which both are alive. Then for any time $t_{1} \leq t$ when $p_{2}$ reached line 16 and any time $t_{2} \leq t$ when $p_{2}$ reached line 16, it follows from Lemma 9 that $\underline{\text { trust }}_{p_{1}}$ at time $t_{1}$ and trust $_{p_{2}}$ at time $t_{2}$ intersect.

From Claims 4 to 8, we can deduce the following theorem:

Theorem 3 (Correctness). The Algorithm 3 implements $\mathcal{T} \Sigma^{l r}$ in $\mathcal{M}_{\mathrm{RME}}$.

Corollary 2 (Necessity). The $\mathcal{T} \Sigma^{l r}$ failure detector is necessary to solve the RME in any unknown dynamic environment with partial memory loss.

\section{Conclusion}

In this paper, we redefined the $\left(\mathcal{T}+\Sigma^{l}\right)$ failure detector into the $\mathcal{T} \Sigma^{l r}$ failure detector adapted to unknown dynamic systems with partial memory loss and where faulty processes may recover. We proved that $\mathcal{T} \Sigma^{l r}$ is both necessary and sufficient to solve the RME problem in such systems, and it is therefore the weakest failure detector to solve RME in unknown dynamic systems with partial memory loss.

Additionally, we showed that the properties of $\left(\mathcal{T}+\Sigma^{l}\right)$ can apply to two separate output variables or to a single one, without changing the strength of the failure detector.

We focused on a specific definition of the mutual exclusion problem for crash-recovery, more specifically the variant where processes stay in CS after a temporary crash. It would be interesting to study other definitions, considering, for instance, that temporary crashes make a process to restart from the remainder section, even if it was in the critical section previously. On the other hand, the definition that we adopted in this paper provides stronger properties, and notably ensures that once a process, which is not eventually down, enters the critical section, it does not have to leave it until it decides to.

\section{Acknowledgements}

E. Mauffret was supported by the RainbowFS project, funded by the ANR project ANR-16-CE25-0013 within the program (DS0703) 2016.

D. Jeanneau was supported by the Labex SMART, supported by French state funds managed by the ANR within the Investissements d'Avenir programme under reference ANR-11-LABX-65.

\section{References}

[1] Divyakant Agrawal and Amr El Abbadi. An efficient and fault-tolerant solution for distributed mutual exclusion. ACM Trans. Comput. Syst., 9(1):120, February 1991.

[2] Marcos Kawazoe Aguilera, Wei Chen, and Sam Toueg. Failure detection and consensus in the crashrecovery model. Distributed Computing, 13(2):99$125,2000$.

[3] Vibhor Bhatt, Nicholas Christman, and Prasad Jayanti. Extracting quorum failure detectors. In Proceedings of the 28th Annual ACM Symposium on Principles of Distributed Computing, PODC 2009, pages 73-82, 2009.

[4] Arnaud Casteigts, Paola Flocchini, Walter Quattrociocchi, and Nicola Santoro. Time-varying graphs and dynamic networks. IJPEDS, 27(5):387-408, 2012 .

[5] Tushar Deepak Chandra and Sam Toueg. Unreliable failure detectors for reliable distributed systems. Journal of the ACM, 43(2):225-267, 1996. 
[6] Ye-In Chang, Mukesh Singhal, and Ming T. Liu. A fault tolerant algorithm for distributed mutual exclusion. In Ninth Symposium on Reliable Distributed Systems, SRDS 1990, pages 146-154, 1990.

[7] Carole Delporte-Gallet, Hugues Fauconnier, and Rachid Guerraoui. Tight failure detection bounds on atomic object implementations. JACM, 57(4), 2010 .

[8] Carole Delporte-Gallet, Hugues Fauconnier, Rachid Guerraoui, and Petr Kouznetsov. Mutual exclusion in asynchronous systems with failure detectors. Journal of Parallel and Distributed Computing, 65(4):492-505, apr 2005.

[9] E. W. Dijkstra. Solution of a problem in concurrent programming control. Communications of the ACM, 8(9):569, 1965.

[10] Michael J. Fischer, Nancy A. Lynch, and Mike Paterson. Impossibility of distributed consensus with one faulty process. J. ACM, 32(2):374-382, 1985.

[11] Wojciech M. Golab and Danny Hendler. Recoverable mutual exclusion in sub-logarithmic time. In Proceedings of the ACM Symposium on Principles of Distributed Computing, PODC 2017, pages 211-220, 2017.

[12] Wojciech M. Golab and Aditya Ramaraju. Recoverable mutual exclusion: [extended abstract]. In Proceedings of the 2016 ACM Symposium on Principles of Distributed Computing, PODC 2016, pages 65-74, 2016.

[13] Prasad Jayanti and Anup Joshi. Recoverable FCFS mutual exclusion with wait-free recovery. In 31st International Symposium on Distributed Computing, DISC 2017, pages 30:1-30:15, 2017.

[14] S. Nishio, E. Manning, and K. Li. A resilient mutual exclusion algorithm for computer networks. IEEE Transactions on Parallel and Distributed Systems, 1:344-356, 071990.

[15] Thibault Rieutord, Luciana Arantes, and Pierre Sens. Détecteur de défaillances minimal pour le consensus adapté aux réseaux inconnus. In Algotel, 2015.
[16] Julien Sopena, Luciana Bezerra Arantes, and Pierre Sens. Performance evaluation of a fair fault-tolerant mutual exclusion algorithm. In 25th IEEE Symposium on Reliable Distributed Systems (SRDS 2006). 